
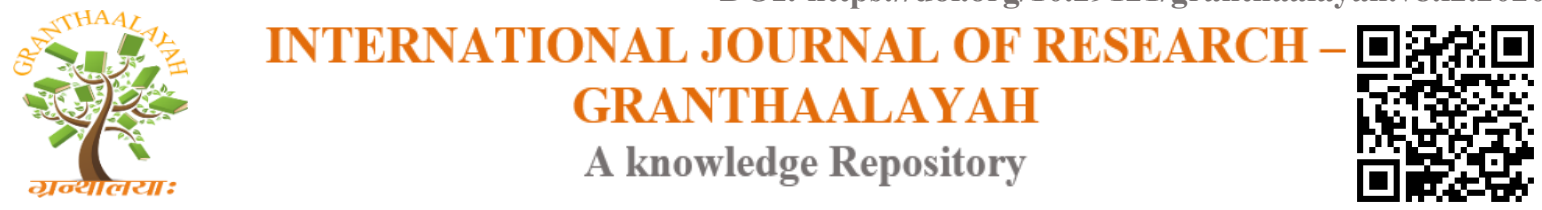

Science

\title{
THE EFFECT OF 500 NM DOSAGE OF NIMODIPINE ON NRF2 AND NF- KB EXPRESSION IN NEURON CELL LINE CULTURE SH-SY5Y EXPOSED WITH CHRONIC HYPERGLYCEMIA
}

\author{
Shahdevi Nandar Kurniawan *1, Muthia Marwah ${ }^{1}$, Rodhiyan R ${ }^{1}$, Nanik $\mathbf{S}^{2}$ \\ ${ }^{* 1}$ Neurology Department, Faculty of Medicine Brawijaya University, Malang, Indonesia \\ ${ }^{2}$ Public Health Department, Faculty of Medicine Brawijaya University, Malang, Indonesia
}

\begin{abstract}
Introduction: Metabolic stress and mitochondrial dysfunction in chronic hyperglycemia can cause an increase in $\mathrm{Ca}^{2+}$ cytosol ions that cause dysfunction of NRF2, that will increase the activity of NF-kB which causes an increase in cytokine production, where NF-kB can modulate NRF2 transcription and its activity, so that it has positive or negative effects in the target gene. Giving nimodipine in chronic hyperglycemia conditions can increase levels of NRF2 molecules and reduce levels of NF-kB. Aim: The purpose of this research is to know the effect of treating nimodipine dose $5 \mu \mathrm{M}$ towards expression of the protein NRF2and NF-kB in SH-SY5Y cell culture induced by chronic hyperglycemia.

Method: This study is a true experimental study using randomized posttest only controlled group design in human SH-SY5Y neuron cell culture. Neuron cells were exposed with chronic glucose (25 mM / G25 normoglycemia, $50 \mathrm{mM}$ / G50 hyperglycemia) for 6 days, followed by administration of nimodipine (without nimodipine and nimodipine $500 \mathrm{nM}$ ) for 30 minutes. Calculation of NRF2 and NF-kB expressions done by fluorescence method.

Results: There were significant differences in the NF-kB neuron cells in the treatment group with controls, both normoglycemia and hyperglycemia ( $\mathrm{p}<0.05)$. Nimodipine was not able to increase the expression of NRF-2 in the treatmnrnt group, although there were significance differences between these two groups. The Spearman Rank correlation test showed a negative correlation between NRF2 and NF-kB expression in the SH-SY5Y human cell line neuron culture that exposed to chronic hyperglycemia with administration of $500 \mathrm{nM}$ nimodipine.

Conclusion: There was a statistically significant decrease in NF-kB expression in SH-SY5Y neuron cells that exposed to chronic hyperglycemia given nimodipine compared to controls group. Nimodipine treatment to SH-SY5Y neuron cells exposed to hyperglycemia not yet increase expression of NRF2.
\end{abstract}

Keywords: Nimodipine; NRF2; NF-kB; Hyperglycemia; SH-SY5Y.

Cite This Article: Shahdevi Nandar Kurniawan, Muthia Marwah, Rodhiyan R, and Nanik S. (2020). "THE EFFECT OF 500 NM DOSAGE OF NIMODIPINE ON NRF2 AND NF-KB EXPRESSION IN NEURON CELL LINE CULTURE SH-SY5Y EXPOSED WITH CHRONIC 
HYPERGLYCEMIA.” International Journal of Research - Granthaalayah, 8(2), 235-242. https://doi.org/10.29121/granthaalayah.v8.i2.2020.214.

\section{Introduction}

According to the American Diabetes Association (ADA) 2005, Diabetes mellitus (DM) is a group of metabolic diseases characterized by hyperglycemia that occurs due to abnormalities in insulin secretion, action of insulin or both. WHO estimates that Indonesia will become the fifth country in worldwide with diabetes as many as 12.4 million people in 2025, get higher 2 levels compared to 1995 (Sudoyo AW, Setiyohadi B, Alwi I, Simadibrata M, 2009). In general, the main complications of DM involve macrovascular as well as damage to peripheral blood vessels, stroke and cardiovascular disorders, where microvascular complications include diabetes retinopathy, diabetes nephropathy, diabetes neuropathy and microangiopathy (Negi and Jena, 2019).

In diabetes mellitus, metabolic stress occurs and mitochondrial dysfunction result in deregulation of calcium ion hemostasis and calcium signaling, which cause pathological cellular reactions that underlie the development of diabetic neuropathy. This disruption of hemostasis causes a steady increase in the concentration of $\mathrm{Ca}^{2+}$ cytosol ions, and a decrease in the amplitude of the $\mathrm{Ca}^{2+}$ ion depolarization induction signal (Verkhratsky and Fernyhough, 2014). Whereas oxidative stress in peripheral nerves causes hyperexcitability in afferent nociceptors and central neurons which leads to the formation of spontaneous impulses in axons and dorsal ganglion roots of the nerve that causes diabetic neuropathy (Oyenihi, et.al., 2015).

The occurrence of oxidative stress triggers the activation of redox regulated transcription factors such as NRF2, and NF-KB. In hyperglycemia stress conditions, extracelullar related kinase (ERK) activation occurs which is inhibiting NRF2 activation. Decreased NRF2 activity and increased NF$\kappa \mathrm{B}$ activity can trigger neuroinflamation and increase oxidative / nitrosative stress. This condition in the future will lead to accumulation of damage to peripheral nerve fibers, and the development of neuropathic pain (Russell et al., 2002).

On the other hand, effective treatment of diabetes neuropathy is currently based on the AAN (American Academy of Neurology), its effectiveness is still not optimal (Neurology, 2007). By looking at the potential for haemostatic disorders that cause increased amount of calcium cytosol, calcium channel blocker (CCB) administration, one of which is nimodipine can be a therapeutic target in improving complications especially in diabetic neuropathy by increasing levels of the NRF2 molecule as a proinflammatory protein and NF-kB as an anti-inflammatory protein. Nimodipine plays a role in the mobilization of calcium, which is the entry of calcium through $\mathrm{L}$ type calcium channels. According to a study by Allen et al in 1983, it is known that nimodipine, $\mathrm{Ca}^{2+}$ antagonist drug has a relaxing effect on brain blood vessels in animal models and neutralizes vasospasm in humans.

Nimodipine increases the sciatic NBF of rats with diabetic neuropathy compared to those treated with a placebo even though it does not restore NBF (Nerve Blood Flow) to a level that is in control / normal. The effect of nimodipine on NBF may be due to the local vasodilator effect because it has very little effect on measured systemic blood pressure. Nimodipine prevents the entry of $\mathrm{Ca}^{2+}$ 
in vascular smooth muscle which will cause reduced blood vessel tone and ultimately local blood flow repair (Kappelle et al., 1994).

To find out the relationship between calcium channel blocker (nimodipine) and NRF2 and NF-kB protein levels in diabetic neuropathy, initial research is needed that uses cells that represent dorsal root ganglion (DRG) neurons, namely SH-SY5Y exposed to high glucose concentrations, with various doses between $10 \mathrm{mM}$ to hyperglycemia of $45 \mathrm{mM}$ (Vetter and Lewis, 2010).

\section{Materials and Methods}

\subsection{Research Design}

This study uses a pure experimental research design (true experimental design) at the Central Laboratory of Biological Sciences (LSIH) Universitas Brawijaya in vitro on neuron cell culture.

\subsection{Research Samples}

The sample of this study used SH-SY5Y culture cells that met the inclusion and exclusion criteria, with a total sample of 16 . Samples were divided into 4 groups namely normoglycemia/G25 without nimodipine $(\mathrm{n}=4)$, normoglycemia/G25 with nimodipine $500 \mathrm{nM}(\mathrm{n}=4)$, hyperglycemia/G50 without nimodipine (n-4), and hyperglycemia/G50 with nimodipine $(n=4)$

\subsection{NRF2 and NF-kB Expression Examination}

Examination of NRF2 and NF-kB expression using antibodies by immunoflorescence. NRF2 and NF-kB observations were carried out using confocal laser screening microscopy (CLSM). NRF2 and NF-kB calculation is done with Olympus Fluoview Ver4.2a with an au unit (aberrant unit).

\section{Results}

Influence of Nimodipin on NRF2 Expression on SH-SY5Y Neuronal Cells Expressed in Chronic Hyperglycemia

One way ANNOVA different test was conducted to find out whether the effect between treatments on NRF2 expression was statistically significantly different. In this test data normality requirements are met. NRF2 variable normality test results showed $p$ value $<0.05$, the assumption of normality was not met. Homogeneity test results showed $\mathrm{p}$ value $<0.05$, the assumption of homogeneity was not fulfilled. The research data was then transformed by the Ln transformation.

The transformed NRF2 variable shows $\mathrm{p}$ value> 0.05 , the assumption of normality is fulfilled. Homogeneity test results showed p value $>0.05$, homogeneity assumptions were met. One Way ANOVA test results showed that there were significant differences in the mean intracellular calcium of the four treatment groups ( $\mathrm{p}<0.05$ ), so that further LSD (Least Significance Difference) tests were performed. There were differences in the mean NRF2 levels in the normoglycemia group + Nimodipine $500 \mathrm{nM}$ with hyperglycemia and control hyperglycemia with hyperglycemia + Nimodipine $500 \mathrm{nM}$. 


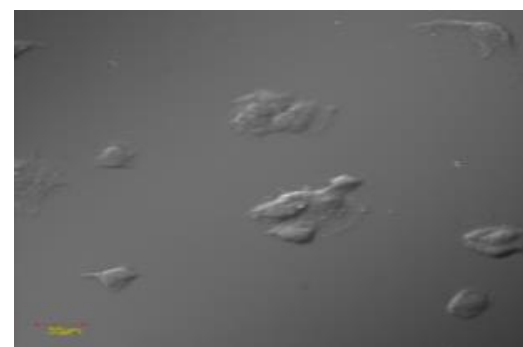

A.

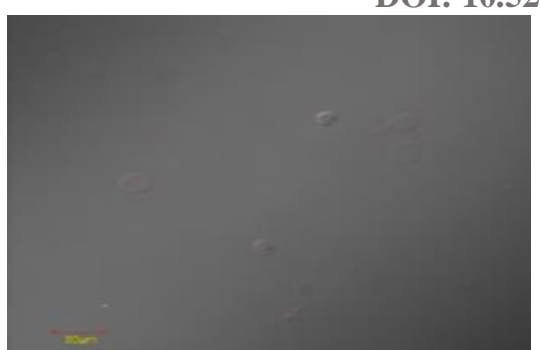

B.

Figure 1: NRF2 expression in neuron culture by treating hyperglycemia $(50 \mathrm{mM})$. Figures $A$ without the administration of nimodipine, B are treated with $500 \mathrm{nM}$ nimodipine. (Culture day 6 , NRF2 red rhodamine, DIC, superimpose, 400x magnification).

The highest average NRF2 expression was found in the normoglycemic treatment given nimodipine which was 33.76 AU, slightly higher than the normoglycemic control group without nimodipine which was 17.66 AU. High NRF2 expression in neuron cells appears as a brighter red color around the cell, as in Figure 1. Superimposed images represent images of cells with fluorescence around them. Whereas the fluorescence lizard represents a picture of the cell's density expressing NRF2, by suppressing the object of the neuron cell. Figure 1 shows the expression of NRF2 in SH-SY5Y neurons cells given either nimodipine or without nimodipine as a result of glucose uptake.

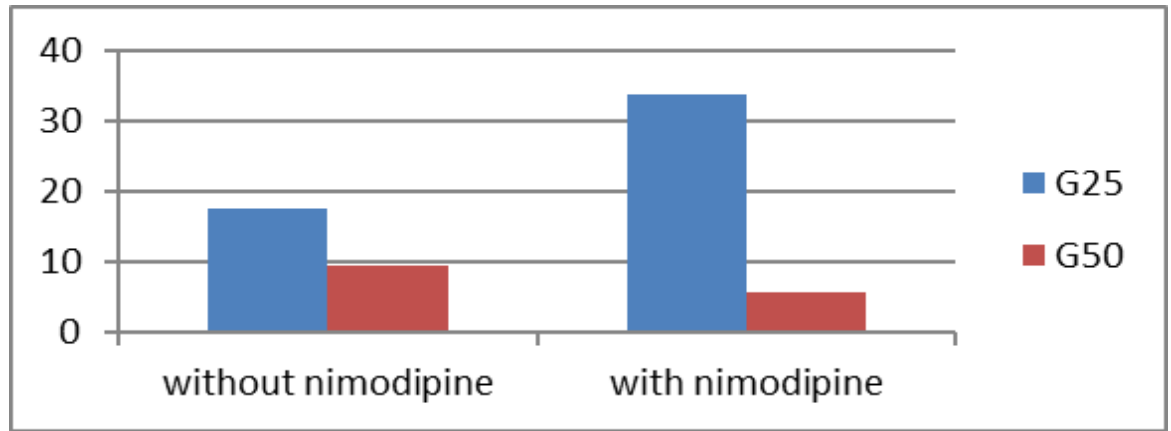

Figure 2: Average histogram of NRF2 expression in SH-SY5Y neuron culture with nimodipine $500 \mathrm{nM}$ intervention and without nimodipine. G25, glucose $25 \mathrm{mM}$; G50, glucose $50 \mathrm{mM}$.

Influence of Nimodipin on NF-kB Expression in SH-SY5Y Neuronal Cells Expressed in Chronic Hyperglycemia

One Way ANOVA test results showed that there were significant differences in the mean NF-kB of the four treatment groups ( $p<0.05$ ), so that further LSD (Least Significance Difference) tests were performed. There were differences in the mean levels of NF-kB in the normoglycemic group with hyperglycemia and hyperglycemia with hyperglycemia + Nimodipine $500 \mathrm{nM}$. The average NF-kB expression in the normoglycemic control group without nimodipine (73.20 AU), was slightly lower than the normoglycemic group given nimodipine (84.03 AU). Whereas in the control group hyperglycemia control (177.39 AU), the average expression was higher than the expression of cells treated with hyperglycemia with nimodipine (549.94 AU). The highest NF- $\mathrm{kB}$ expression in neuron cell culture appears as a brighter red color around the cell, as presented in Figure 2. The brightest red color appears in the hyperglycemia control treatment. 


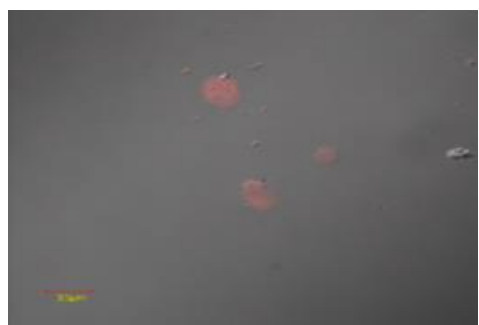

A.

Figure 3: NF-kB expression in neuron culture by treating hyperglycemia $(50 \mathrm{mM})$. Figures $\mathrm{A}$ without the administration of nimodipine, B are treated with $500 \mathrm{nM}$ nimodipine. (Culture day 6 , NF-kB red rhodamine, DIC, superimpose, 400x magnification).

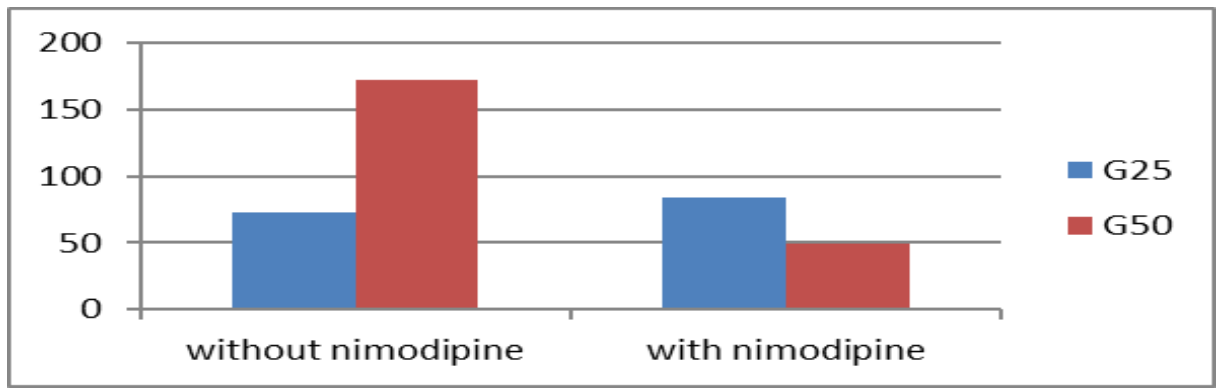

Figure 4: Average histogram of NF-kB expression in SH-SY5Y neuron culture with nimodipine $500 \mathrm{nM}$ intervention and without nimodipine. G25, glucose $25 \mathrm{mM}$; G50, glucose $50 \mathrm{mM}$.

Correlation of NRF2 expression and NF- $\kappa$ B expression on SH-SY5Y neuron cells in hyperglycemia conditions

The correlation coefficient is a quantity that states the closeness of the linear relationship between two variables. In this study, we want to know how closely the relationship between NRF2 and NF$\mathrm{kB}$ in SH-SY5Y neuron cells with nimodipine, which is treated with hyperglycemia, uses the Spearman Rank correlation test. From the results of the correlation test, the value of $\mathrm{p}<0.05$ showed that the correlation between NRF2 and NF-kB levels in hyperglycemia conditions with nimodipine was significant. The correlation coefficient (r) of -0.913 indicates a negative correlation. These results indicate that the higher the NRF2, the lower the NF-kB level.

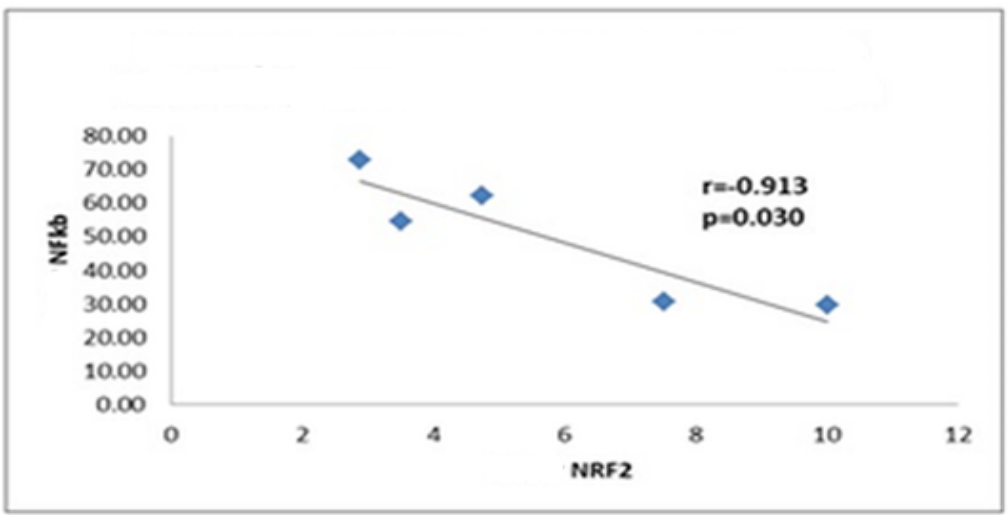

Figure 5: The correlation test of NRF2 levels and NF-kB levels under hyperglycemia conditions with nimodipine $500 \mathrm{nM}$. 


\section{Discussion}

\section{Expression of NRF2 and NF-kB in SH-SY5Y Neuron Cells Expressed in Chronic Hyperglycemia}

NRF2 is a bZIP protein (basic leucine zipper) whose main function is the main defense mechanism against cellular oxidative stress. NRF2 maintains hemostasis under stress conditions (Kumar and Mittal, 2017). NRF2 is involved in the expression of various antioxidant proteins (such as detoxification enzymes) through the antioxidant response element (ARE) binding site. Under normal conditions, NRF2 is inactive and is located in the cytosol (Kumar and Mittal, 2017). NRF2 will be activated during stress / increase in free radical production. NRF2, which actively migrates into the cell nucleus, binds to DNA in elements of the antioxidant response and decreases the production of free radicals and oxidative stress. NRF2 will be inactive in the cytosolic compartment, along with its binding protein, Keap2. Under conditions of oxidative stress, Keap1 releases NRF2, so that NRF2 becomes active, goes to the nucleus and activates transcription genes for antioxidants (Schroeder et al., 2014). NRF2 activator can be one of the managements of diabetes neuropathy in the future (Kumar and Mittal, 2017).

In this study it was found that NRF2 expression decreased in chronic hyperglycemia conditions by administering nimodipine and its value was statistically significant. In accordance with previous theories that, in the early stages of hyperglycemia, NRF2 signals act as the main regulator to induce cytoprotective genes, so their levels increase. However, in cases of persistent hyperglycemia, NRF2 levels decrease resulting in progression of complications (neuropathy) through the neuroinflamation pathway (Jiang et al., 2010, Kumar and Mittal, 2017). Activation of NRF2 will suppress hyperglycemia which induces oxidative stress and metabolic dysfunction in human endothelial cells. Whereas down regulation of NRF2 will cause various microvascular changes, which cause diabetic neuropathy (Bhakkiyalakshmi et al., 2015). It was also said that Nrf2 levels were only high in the first 24 - 48 hours during hypoxic exposure (Schroeder et al., 2014)

NF-kB expression is also increased in SH-SY5Y neuron cells exposed to chronic hyperglycemia. $\mathrm{NF}-\kappa \mathrm{B}$ is activated by oxidative stress due to hyperglycemia conditions and AGE products. NF$\kappa \mathrm{B}$ plays an important role in mediating the inflammatory response. Activation of NF- $\kappa \mathrm{B}$ can induce proinflammatory responses, endothelin regulation, and apoptosis. In SH-SY5Y cells, NF$\kappa \mathrm{B}$ is needed for neuronal differentiation. The increased activation of NF- $\kappa \mathrm{B}$, in response to TNF$\alpha$, is obtained at high glucose concentrations, indicating a potential association with diabetic complications. NF- $\kappa \mathrm{B}$ activation is also found in high glucose Schwan cell cultures compared to low glucose (Petegem \& Minor, 2006; Aristidis \& Rayaz, 2007; Cao et al., 2012).

In this study treatment was given in the form of a $500 \mathrm{nM}$ nimodipin dose, this dose was chosen based on a previous research dose by Shamash,1998. In this study it was also found that the expression of NF-kB decreased after administration of nimodipin. Research conducted by Cao, et al found that retinal pericinal cells showed activation of NF- $\mathrm{NB}$ under hyperglycemia conditions both in vivo and in vitro. The relationship of NF- $\mathrm{KB}$ pericis with the death of microvascular cells and acellular capillaries on the retina, it was found that the activation of NF- $\kappa \mathrm{B}$ induced by diabetes has proapoptotic consequences. The basal activity of $\mathrm{NF}-\kappa \mathrm{B}$ in BRP (Bovine Retinal Pericytes) culture acts as a survival process while its activation during hyperglycemia occurs as a proapoptotic. NF- $\kappa$ B activation increases significantly in glucose $30 \mathrm{mM}$ and increases in glucose 
50 and $100 \mathrm{mM}$ glucose in mesangial cells as in vascular cells. ROS produced by glucose metabolism has an important role in the activation of NF- $\mathrm{KB}$ induced by hyperglycemia in mesangial cells (Ha, Yu, Choi, Kitamura, \& Lee, 2002; Cao et al., 2012).

The results of the Spearman Rank correlation test in this study found a negative correlation between NRF2 expression and NF-kB under hyperglycemia conditions with nimodipine administration. So it can be interpreted that, an increase in NRF2 expression will be accompanied by a decrease in NF-kB expression. This supports the study of literature in 2015. In the study it was said that the reduction of NRF2 will increase the activity of NF-kB which causes an increase in cytokine production, where NF-kB can modulate NRF2 transcription and its activity, so that it has both positive and negative effects on genes target. NRF2 activation is said to inhibit NF-kB activation, and conversely NF-kB is known to inhibit NRF2 (Wardyn et al., 2015).

\section{Conclusions}

There was a statistically significant decrease in NF-kB expression in SH-SY5Y neuron cells exposed with chronic hyperglycemia treated with nimodipin $500 \mathrm{nM}$, compared with SH-SY5Y neuron cells exposed to chronic hyperglycemia without nimodipin. Nimodipin treated in SHSY5Y neuron cells exposed with hyperglycemia not yet increase expression of NRF2. Though correlation test increase in NRF2 expression was accompanied by a decrease in NF-kB expression in SH-SY5Y neuronal cells exposed to chronic hyperglycemia.

\section{Acknowledgement}

This research was carried out with the financial support of BPPM (Agency for Research and Community Service) Faculty of Medicine, Brawijaya University, Malang, Indonesia.

\section{References}

[1] Bhakkiyalakshmi E, Sireesh D, Rajaguru P, Paulmurugan R, Ramkumar KM. The emerging role of redox-sensitive Nrf2-Keap1 pathway in diabetes. Pharmacol Res, 2015, 91:104-114.

[2] Jiang, T. et al. The protective role of Nrf2 in STZ-induced diabetic nephropathy. Progress in physiology. 2010, 41(6), pp. 435-438.

[3] Kappelle, A. C. et al. Beneficial effect of the $\mathrm{Ca} 2+$ antagonist, nimodipine, diabetic neuropathy in the BB / Wor rat existing. 1994, pp. 887-893.

[4] Kumar, A. and Mittal, R. Nrf2: a potential therapeutic target for diabetic neuropathy, Inflammopharmacology. Springer International Publishing. 2017, 25(4), pp. 393-402.

[5] Ma, Q. HHS Public Access, Annual Review of Pharmacology and Toxicology. 2015, (1), pp. 401426.

[6] Negi, C. K. and Jena, G. Nrf2, a novel molecular target to reduce type 1 diabetes associated secondary complications: The basic considerations, European Journal of Pharmacology. Elsevier B.V. 2019, 843, pp. 12-26.

[7] Neurology, A. A. Treatment of painful neuropathy, 2007, pp. 531-535.

[8] Oyenihi, A. B. et al. Antioxidant strategies in the management of diabetic neuropathy, BioMed Research International. Hindawi Publishing Corporation, 2015.

[9] Russell, J. W. et al. High glucose-induced oxidative stress and mitochondrial dysfunction in neurons, FASEB Journal. 2002, 16, p. 1742.

[10] Schroeder, T., Hamilton, K. and Irwin, D. C. NIH Public Access. 2014, pp. 264-273. 
[11] Sudoyo AW, Setiyohadi B, Alwi I, Simadibrata M, S. S. Buku Ajar Ilmu Penyakit Dalam. jilid II. Jakarta: Interna Publishing. 2009.

[12] Verkhratsky, A. and Fernyhough, P. Calcium signalling in sensory neurones and peripheral glia in the context of diabetic neuropathies, Cell Calcium. Elsevier Ltd, 2014, 56(5), pp. 362-371.

[13] Vetter, I. and Lewis, R. J. (2010) 'Characterization of endogenous calcium responses in neuronal cell lines', Biochemical Pharmacology, 79(6), pp. 908-920. doi: 10.1016/j.bcp.2009.10.020.

[14] Vinik, A. et al. Diabetic neuropathies: Clinical manifestations and current treatment options, Nature Clinical Practice Endocrinology and Metabolism. 2006, 2(5), pp. 269-281.

[15] Uguz A. C., Naziroglu M., Espino J., Bejarano I., Gonzalez D., Rodri- guez A. B. and Pariente J. A. Selenium modulates oxidative stress-induced cell apoptosis in human myeloid HL-60 cells through regulation of calcium release and caspase-3 and -9 activities. J. Membr. Biol. 2009, 232, $15-23$.

[16] Zhang, D. D. et al. The Nrf2/Keap1/ARE Pathway and Oxidative Stress as a Therapeutic Target in Type II Diabetes Mellitus, Molecular and cellular biology. 2017, 7(11), pp. 769-789.

\footnotetext{
*Corresponding author.

E-mail address: shahdevinandar@ub.ac.id
} 